TRANSACTIONS OF THE

AMERICAN MATHEMATICAL SOCIETY

Volume 349, Number 11, November 1997, Pages 4481-4499

S $0002-9947(97) 02044-8$

\title{
NONSELFADJOINT OPERATORS GENERATED BY THE EQUATION OF A NONHOMOGENEOUS DAMPED STRING
}

\author{
MARIANNA A. SHUBOV
}

\begin{abstract}
We consider a one-dimensional wave equation, which governs the vibrations of a damped string with spatially nonhomogeneous density and damping coefficients. We introduce a family of boundary conditions depending on a complex parameter $h$. Corresponding to different values of $h$, the problem describes either vibrations of a finite string or propagation of elastic waves on an infinite string. Our main object of interest is the family of nonselfadjoint operators $A_{h}$ in the energy space of two-component initial data. These operators are the generators of the dynamical semigroups corresponding to the above boundary-value problems. We show that the operators $A_{h}$ are dissipative, simple, maximal operators, which differ from each other by rank-one perturbations. We also prove that the operator $A_{1}(h=1)$ coincides with the generator of the Lax-Phillips semigroup, which plays an important role in the aforementioned scattering problem. The results of this work are applied in our two forthcoming papers both to the proof of the Riesz basis property of the eigenvectors and associated vectors of the operators $A_{h}$ and to establishing the exact and approximate controllability of the system governed by the damped wave equation.
\end{abstract}

\section{INTRODUCTION}

1.1. Statement of Problem. In this work we study a one parameter family of nonselfadjoint operators naturally related to the wave equation

$$
u_{t t}-\frac{1}{\rho(x)}\left(p(x) u_{x}\right)_{x}+2 d(x) u_{t}+q(x) u=0 .
$$

This equation governs the vibrations of a nonhomogeneous string with the density $\rho(x)$ and modulus of elasticity $p(x)$, subject to a viscous damping with the friction coefficient $2 d(x)$, and affected by an external harmonic force with the rigidity coefficient $q(x)$. We will assume that $x \in[0, \infty)$, i.e. that the string is semi-infinite, and that $u(x, t)$ satisfies the Dirichlet boundary condition at $x=0$ :

$$
u(0, t)=0 .
$$

Both assumptions are made only for convenience of the exposition. All of the analysis below can be extended in a straightforward manner both to the case of an infinite string $x \in \mathbb{R}$ and to more general boundary conditions at $x=0$ (see below).

Received by the editors August 21, 1995 and, in revised form, October 15, 1995.

1991 Mathematics Subject Classification. Primary 47A10; Secondary 47A55, 47B44.

(C)1997 American Mathematical Society 
We assume that the coefficients in Eq. (1.1) satisfy the following conditions:

a) $\rho(x)=p(x)=1, d(x)=q(x)=0$ for $x \in[a, \infty), \quad a>0$;

b) $\rho(x)>0, \quad \infty>\beta \geq p(x) \geq \alpha>0, d(x)>0, q(x) \geq 0, \quad x \in[0, a)$;

c) $\rho, p \in C^{2}[0, a), \quad d \in H^{1}(0, a), \quad q \in \mathbb{L}^{2}(0, a)$,

where $H^{1}$ is the Sobolev space of functions which belong with their first weak derivatives to the space $L^{2}(0, a)$, and we denote by $\mathbb{L}^{2}(0, a)$ the weighted space with the norm

$$
\begin{aligned}
\|\varphi\|_{\mathbb{L}^{2}}^{2} & =\int_{0}^{a}|\varphi(x)|^{2} \rho(x) / p(x) d x ; \\
\text { d) } 0<\mathcal{M} & =\int_{0}^{a} \sqrt{\rho(x) / p(x)} d x<\infty, \\
0<\mathcal{N} & =\int_{0}^{a} d(x) \sqrt{\rho(x) / p(x)} d x<\infty .
\end{aligned}
$$

Remark 1.1. 1) The condition (1.3) means that both the nonhomogeneity and the damping are concentrated on a finite interval $[0, a)$. Depending on the boundary condition at the point $x=a$, which we introduce below, Eq. (1.1) describes either the vibrations of a finite string of length $a$ or the scattering of elastic waves on a semi-infinite string by an obstacle concentrated on $[0, a)$.

2) The last condition (1.4) is introduced for convenience only. It can be replaced by the weaker restriction $q(x) \geq-C, C>0$.

3) It is clear from (1.4) - (1.7) that the function $\rho$ may have zero or singularity at $x=a$. In fact, the restrictions on both $\rho$ and $p$ may be weakened. We only need that $\rho(x) \geq 0, p(x) \geq 0$ and (1.7) to be satisfied, i.e. both functions may have zeros and singularities on the interval $[0, a)$. Also instead of the first condition in (1.5), we can assume that both $\rho$ and $p$ have first and second order weak derivatives which belong to $L^{2}(0, b)$ for any $b<a$. The above stronger restrictions are introduced only for simplification of the exposition.

Now we introduce a one-parameter family of boundary conditions at $x=a$ :

$$
u_{x}(a, t)+h u_{t}(a, t)=0, \quad t \in[0, \infty) .
$$

where $h \in \mathbb{C} \cup\{\infty\}$ is a complex parameter with $R e h \geq 0$. To $h=\infty$ we formally associate the condition

$$
u_{t}(a, t)=0,
$$

which is equivalent to the Dirichlet boundary condition $u(a, t)=$ const. To have an initial-boundary problem it remains to introduce the initial conditions

$$
u(x, 0)=u_{0}(x), u_{t}(x, 0)=u_{1}(x), x \in[0, \infty) .
$$

The restrictions on $u_{0}$ and $u_{1}$ will be given below.

Let us mention some particular cases of the problem (1.1), (1.2), (1.8), (1.10) corresponding to different values of the parameter $h$. In the case $h=0$ we have the Neumann boundary condition at $x=a$, and our problem describes the vibrations of a finite string with one end fixed and the other free. In the case $h=\infty$ the condition (1.8) turns into the Dirichlet boundary condition (1.9), and we have a finite string with both ends fixed. In both cases, the only region of interest is $[0, a]$ 
and we need to know the initial data (1.10) only for $x \in[0, a]$. If $h=1$, then (1.8) is the so-called Sommerfeld radiation boundary condition. In this case our problem describes the scattering of elastic waves on the semi-infinite string $[0, \infty)$ by an inhomogeneity of the medium concentrated on the interval $[0, a]$ (see [12]).

Our main object of interest in this work is a one-parameter family of nonselfadjoint operators $A_{h}(h \in \mathbb{C} \cup\{\infty\})$ naturally associated to the above problem (1.1), (1.2), (1.8), (1.10). To define these operators, we introduce a matrix differential expression

$$
\mathfrak{L}=-i\left(\begin{array}{cc}
0 & 1 \\
\frac{1}{\rho(x)} \frac{d}{d x}\left(p(x) \frac{d}{d x} \cdot\right)-q(x) . & -2 d(x)
\end{array}\right)
$$

acting on two-component functions $U(x)=\left(\begin{array}{l}u_{0}(x) \\ u_{1}(x)\end{array}\right)$. Using (1.11) we can formally rewrite the problem (1.1), (1.2), (1.8), (1.10) in the form

$$
\begin{gathered}
U_{t}=i \mathfrak{L} U, \\
u_{0}(0, t)=0, \quad u_{0 x}(a, t)+h u_{1}(a, t)=0, \\
U(x, 0)=U_{0}(x)=\left(\begin{array}{c}
u_{0}^{0}(x) \\
u_{1}^{0}(x)
\end{array}\right), x \in[0, \infty),
\end{gathered}
$$

where $U(x, t)=\left(\begin{array}{l}u_{0}(x, t) \\ u_{1}(x, t)\end{array}\right)$ and $u_{0}=u$ is exactly the unknown function from (1.1).

Let us consider the space of smooth two-component functions $U(x)=\left(\begin{array}{l}u_{0}(x) \\ u_{1}(x)\end{array}\right)$ (initial data) defined on the interval $x \in[0, a]$ such that $u_{0}$ vanishes in the vicinity of $x=0$. The closure of this space in the energy norm

$$
\|U\|_{\mathcal{H}_{a}}^{2}=\frac{1}{2} \int_{0}^{a}\left(p(x)\left|u_{0}^{\prime}(x)\right|^{2}+q(x) \rho(x)\left|u_{0}(x)\right|^{2}+\rho(x)\left|u_{1}(x)\right|^{2}\right) d x
$$

is a Hilbert space, which we denote by $\mathcal{H}_{a}$. We define $A_{h}$ as the linear operator in $\mathcal{H}_{a}$ given by the differential expression (1.11), i.e., $A_{h}=\mathfrak{L}$ on the domain

$$
D\left(A_{h}\right)=\left\{U \in \mathcal{H}_{a}: u_{0}^{\prime \prime}, u_{1}^{\prime} \in \mathbb{L}^{2}(0, a), u_{1}(0)=0, \quad u_{0}^{\prime}(a)+h u_{1}(a)=0\right\} .
$$

Notice that problem (1.12) - (1.14) defines an evolution $C_{0}$-semigroup in the space $\mathcal{H}_{a}$. The operator $i A_{h}$ is exactly the generator of this semigroup.

Remark 1.2. a) The condition $u_{1}(0)=0$ in (1.16) requires an explanation. Notice that the boundary condition $u_{0}(0)=0$ is satisfied automatically due to the definition of the space $\mathcal{H}_{a}$. Recall that $u_{0 t}(x, t)=u_{1}(x, t)$ due to (1.1). This relation together with the first condition (1.13) implies that $u_{1}(0, t)=0$. Therefore, the stationary variant of the latter condition must be included in the definition of $D\left(A_{h}\right)$.

b) If $\rho$ has no singularities or zeros, then the condition $u_{0}^{\prime \prime}, u_{1}^{\prime} \in \mathbb{L}^{2}(0, a)$ in (1.16) is equivalent to the requirement that $u_{0} \in H^{2}(0, a), u_{1} \in H^{1}(0, a)$. (Here we use the standard notation $H^{k}(0, a)$ for Sobolev spaces.) 
Our main results are contained in the four theorems concerning the operators $A_{h}$, of which three (Theorems 2.1-2.3) will be stated in Section 2 and one (Theorem 4.2 ) will be given in Section 4 . We briefly describe below these results as a part of the program devoted to the spectral analysis of the operators $A_{h}$.

1.2. Applications to Spectral Analysis and Control Theory. The present paper, while being a closed piece of work, is, in fact, one in a series of papers by the author [18]-[24]. These works, together with two forthcoming papers, are devoted to the spectral analysis of the above described operators $A_{h}$ (and the generalizations of these operators related to 3-dimensional spherically symmetric nonhomogeneous damped wave equation) and to applications of the spectral results to the control theory of distributed parameter systems. In order to explain the significance of the results of the present work, we give below a brief description of the whole program, which is partially carried out in the aforementioned papers and will be completed in the forthcoming works.

As is well known, at the present time there is no general spectral theory of nonselfadjoint operators in a Hilbert space. Such a theory has been constructed only for some special classes of nonselfadjoint operators [3]-[5]. One such class is the class of spectral operators [3]. While the general abstract theory of such operators has been developed a long time ago, there is still a problem of finding specific examples of the operators of this class. In particular, it turned out that for many natural nonselfadjoint operators (even of one independent variable) it is very difficult to verify the spectral property.

One of the main results of the above series of works (see $[19,20,22,23])$ is the fact that the operators $A_{h}$ defined in (1.11) and (1.16) provide a class of nontrivial examples of spectral operators. To explain this statement, we recall the definition of a spectral operator. (The definition we give below is a particular case of the general definition [3]. However it is sufficient for our examples.)

Definition 1.1. Let $A$ be a closed unbounded linear operator in a complex Hilbert space $H$ defined on a dense domain $D(A)$. We say that $A$ is spectral if there exists a Riesz basis in $H$ (linear isomorphic image of an orthonormal basis), which belongs to $D(A)$, such that the matrix of the operator $A$ in this basis has a Jordan normal form with only a finite number of nontrivial Jordan blocks (the rest of the matrix is diagonal).

To show that a specific operator $A$ is spectral in the sense of Definition 1.1 it is sufficient to prove the following facts:

(i) A has a discrete spectrum.

(ii) Only a finite number of the eigenvectors of A have finite chains of associated vectors $(\varphi \in H$ is an associated vector of $A$ of order $m$ corresponding to the eigenvalue $\lambda$ if $\left.(A-\lambda I)^{m+1} \varphi=0\right)$.

(iii) The system of eigenvectors and associated vectors of the operator $A$ forms a Riesz basis in $H$.

The properties (i) and (ii) for the operators $A_{h}$ are known. The most difficult part in the proof of the fact that $A_{h}$ is spectral is the proof of the Riesz basis property (iii). We outline below the main steps in the proof of this result. The results of the present work, while being of interest in themselves, form one of these steps. We mention here that in the case of Eq. (1.1) with $p(x)=1, d(x)=0$ and with the boundary conditions (1.2), (1.8) the Riesz basis property result is known 
$[14,18]$. The case of $p(x)=\rho(x)=1, d(x) \neq 0$ with the Dirichlet boundary conditions at both ends $(x=0, x=a)$ was recently studied in [1]. However, the combination of a nonconstant density and damping with nonselfadjoint boundary conditions makes even this 1-dimensional problem very nontrivial from the point of view of spectral analysis.

The first step of the aforementioned program (see $[19,20])$ consists in the derivation of the asymptotic formulas for the eigenvalues $\left\{\lambda_{n}\right\}_{n \in \mathbb{Z}}$ and eigenvectors $\left\{\Phi_{n}\right\}_{n \in \mathbb{Z}}$ of the operator $A_{h}$ as $|n| \rightarrow \infty$. (The spectrum of $A_{h}$ belongs to a strip in the complex plane parallel to the real axis. It has only two limit points: $+\infty$ and $-\infty$. That is why it is convenient to use the index $n \in \mathbb{Z}$.)

As the second step we show that all of the operators $A_{h}(h \in \mathbb{C} \cup\{\infty\})$ differ from the operator $A_{1}(h=1)$ by rank one perturbations. This step is carried out in the present work (Theorem 2.2 and Corollary 2.1).

Combining this result with the asymptotic formulas for the eigenvalues and the eigenvectors, we show in [22] that it is sufficient to prove the Riesz basis property of the root vectors (eigen- and associated vectors) only for $A_{1}$. In general, a rank one perturbation can destroy the Riesz basis property. But, as we show, this is not the case in our problem.

Recall that the case $h=1$ corresponds to the scattering problem on a finite obstacle on the semi-infinite string. The eigenvalues of $A_{1}$ are called resonances and the corresponding eigenvectors are called resonance states. To prove the Riesz basis property for $A_{1}$, we invoke the Lax-Phillips scattering theory [8] (see Section 2 for a brief description of this theory). Our next step (again carried out in this work) is the proof that $A_{1}$ coincides with the generator $\mathcal{B}$ of the so-called Lax-Phillips semigroup (Theorem 2.3).

Our final step [22] consists of the construction of one more element of the LaxPhillips theory: the so-called incoming spectral representation. This is a linear isomorphism $\mathbb{T}$ which maps the energy space $\mathcal{H}_{a}$ onto a subspace of the Hardy space $H_{+}^{2}[6] .\left(H_{+}^{2}\right.$ is the space of all $L^{2}(\mathbb{R})$-functions having analytic continuation to the upper half-plane.) $\mathbb{T}$ establishes an equivalence between the generator $\mathcal{B}=A_{1}$ of the Lax-Phillips semigroup and the so-called model "shift operator $S$ " in $H_{+}^{2}[4,13]$. Therefore, the problem has been reduced to the proof of the Riesz basis property for $S$. The latter can be easily established based on our asymptotic formulas for $\left\{\lambda_{n}\right\}$ ( $S$ has the same spectrum as $A_{1}$ ) and the Carleson theorem [13].

All of the steps of the above outlined program are carried out in our work [18] for the case of the radial wave equation (which involves a more general Sturm-Liouville operator than (1.1)) with one significant simplification: $d(x)=0$. The presence of a nonzero damping makes the problem much more complicated. The reason for this consists of the following. If $d(x)=0$, then the differential operator $\mathfrak{L}_{0}$ defined by (1.11) and (2.1) is symmetric in $\mathcal{H}_{a}$ (although not maximal). In this case $A_{h}$ would have been nonselfadjoint only due to the boundary condition at $x=a$. This is a relatively "mild" nonselfadjointness. If $d(x) \neq 0$, then $\mathfrak{L}_{0}$ is nonsymmetric in $\mathcal{H}_{a}$.

To conclude this Introduction, we mention that the above Riesz basis property result allows us to solve the so-called controllability problem for the damped string equation [21, 24]. In the late sixties D. Russell [15]-[17] suggested the spectral decomposition method for the solution of this problem in the case of an undamped string: $d(x)=0$. Our results allow us to extend Russell's method to the damped string equation. 


\section{Statement of Main Results}

Before we formulate our main results we need to recall some definitions. ([5, 11, $13])$.

Definition 2.1. A closed linear operator $A$, acting in a Hilbert space $H$ and having domain $D(A)$ dense in $H$ is said to be dissipative if

$$
\operatorname{Im}(A f, f) \geq 0 \text { for } f \in D(A) .
$$

Definition 2.2. A dissipative linear operator $A$ defined on a domain $D(A)$ dense in $H$ is said to be simple if $A$ and $A^{*}$ do not have a common invariant subspace on which they coincide.

Definition 2.3. A dissipative linear operator $A$ in $H$ is said to be maximal if it does not admit any extensions.

Our first main result is given by the following theorem.

Theorem 2.1. The operator $A_{h}$ with $h \in \mathbb{C} \cup\{\infty\}$, Re $h \geq 0$, defined by the differential expression (1.10) on the domain (1.15) is a dissipative, simple, maximal operator in the energy Hilbert space $\mathcal{H}_{a}$.

To formulate our second result we have to introduce an auxiliary operator $\mathfrak{L}_{0}$. This operator acts in the space $\mathcal{H}_{a}$. It is given by the same differential expression (1.10), i.e. $\mathfrak{L}_{0}=\mathfrak{L}$, but has the domain

$$
\begin{array}{r}
D\left(\mathfrak{L}_{0}\right)=\left\{U=\left(\begin{array}{l}
u_{0} \\
u_{1}
\end{array}\right) \in \mathcal{H}_{a}: u_{0}^{\prime \prime}, u_{1}^{\prime} \in \mathbb{L}^{2}(0, a), u_{1} \in H^{1}(0, a) ;\right. \\
\left.u_{1}(0)=u_{0}^{\prime}(a)=u_{1}(a)=0\right\} .
\end{array}
$$

Our second result consists of the following.

Theorem 2.2. $\mathfrak{L}_{0}$ is a dissipative, simple, but not maximal operator. The operators $A_{h}(R e h \geq 0$ or $h=\infty)$ are one dimensional maximal extensions of $\mathfrak{L}_{0}$.

Corollary 2.1. For any $h_{1}, h_{2} \in \mathbb{C} \cup\{\infty\}\left(R e h_{1}\right.$, Re $\left.h_{2} \geq 0\right)$ the difference $A_{h_{1}}-$ $A_{h_{2}}$ is a rank one operator. In particular, all operators $A_{h}$ are rank one perturbations of the operator $A_{1}$.

The significance of the latter result has already been explained in the Introduction.

To formulate our next result we have to recall briefly the Lax-Phillips approach to the scattering theory for a particular case of Eq. (1.1). Consider the problem (1.1), (1.2), (1.10) on the whole semi-axis $x \in[0, \infty)$. In other words, we temporarily exclude from consideration the boundary condition (1.8). This problem describes the propagation of elastic waves on a semi-infinite string $[0, \infty)$ and their scattering on an obstacle, which is concentrated on the interval $[0, a]$ due to our assumptions about $\rho, p, d$ and $q$. Let us define the energy space $\mathcal{H}$ of this problem. Consider the space of smooth two-component initial data $U(x)=\left(\begin{array}{l}u_{0}(x) \\ u_{1}(x)\end{array}\right)$ defined for $x \in[0, \infty)$, such that $u_{0}$ vanishes in the vicinity of $x=0$. 
The closure of this space in the energy norm

$$
\|U\|_{\mathcal{H}}^{2}=\frac{1}{2} \int_{0}^{\infty}\left(p(x)\left|u_{0}^{\prime}(x)\right|^{2}+q(x) \rho(x)\left|u_{0}(x)\right|^{2}+\rho(x)\left|u_{1}(x)\right|^{2}\right) d x
$$

is precisely the energy space $\mathcal{H}$. We notice that, in contrast with $\mathcal{H}_{a}$, the space $\mathcal{H}$ consists of functions defined on $[0, \infty)$, and the only difference between $(2.2)$ and (1.15) is that the upper limit of integration is $\infty$.

We introduce in $\mathcal{H}$ the outgoing and incoming subspaces $\mathcal{D}_{+}$and $\mathcal{D}_{-}$. $\mathcal{D}_{+}$(or $\left.\mathcal{D}_{-}\right)$is the subspace of all initial data such that the corresponding solutions of the initial-boundary problem vanish for $|x|-t \leq a$ (or for $|x|+t \leq a$ ). Denote by $V_{t}$ the evolution operator taking initial data into the solution data at the time $t$. The operators $\left\{V_{t}, t \leq 0\right\}$ form a one-parameter semigroup of contractions in $\mathcal{H}$. (It is convenient for us to take the nonpositive values of $t$.) The subspaces $\mathcal{D}_{+}$and $\mathcal{D}_{-}$ have the following properties [9]:

(i) $V_{t} \mathcal{D}_{-} \subset \mathcal{D}_{-}, \quad V_{t}^{*} \mathcal{D}_{+} \subset \mathcal{D}_{+}$

(ii) $\bigcap_{t \geq 0} V_{t}^{*} \mathcal{D}_{+}=\bigcap_{t \leq 0} V_{t} \mathcal{D}_{-}=\emptyset$.

(iii) Let $P_{+}$and $P_{-}$be the orthogonal projections onto orthogonal complements of $\mathcal{D}_{+}$and $\mathcal{D}_{-}$respectively. Then for each $f \in \mathcal{H}$

$$
\begin{aligned}
& \lim _{t \rightarrow \infty} P_{+} V_{t}^{*} f=0, \\
& \lim _{t \rightarrow-\infty} P_{-} V_{t} f=0 .
\end{aligned}
$$

(iv) $V_{t}$ is an isometry on $\mathcal{D}_{-}$and $V_{t}^{*}$ is an isometry on $\mathcal{D}_{+}$.

Consider the subspace

$$
K=\mathcal{H} \ominus\left(\mathcal{D}_{+} \oplus \mathcal{D}_{-}\right),
$$

which is called the spectral invariant subspace of the problem. Let $P_{K}$ be the orthogonal projector of $\mathcal{H}$ onto $K$. The operators

$$
Z_{t}=\left.P_{K} V_{t}\right|_{K}
$$

form a strongly continuous semigroup of contractions defined on $K$. This is precisely the Lax-Phillips semigroup mentioned in our Introduction. Let $i \mathcal{B}$ be the infinitesimal generator of $Z_{t}$ :

$$
Z_{t}=\exp (i \mathcal{B} t)
$$

$\mathcal{B}$ is an unbounded nonselfadjoint operator with a pure point spectrum $\left\{\lambda_{n}\right\}_{n=-\infty}^{\infty}$.

Now we formulate the statement describing the subspaces $\mathcal{D}_{+}, \mathcal{D}_{-}, K$. The proof can be found in [18].

Lemma 2.1. The subspaces $\mathcal{D}_{+}$and $\mathcal{D}_{-}$can be explicitly described as follows:

$$
\begin{aligned}
& \mathcal{D}_{-}=\left\{U \in \mathcal{H}: \operatorname{supp} U \subset[a, \infty), u_{0} \in H^{1}(a, \infty), u_{1}=u_{0}^{\prime}\right\}, \\
& \mathcal{D}_{+}=\left\{U \in \mathcal{H}: \operatorname{supp} U \subset[a, \infty), u_{0} \in H^{1}(a, \infty), u_{1}=-u_{0}^{\prime}\right\} .
\end{aligned}
$$

The subspace $K$ consists of all data $U \in \mathcal{H}$ such that $u_{1}(x)=0$ for $x \geq a$ and $u_{0}(x)=u_{0}(a)$ for $x \geq a$. This means that $K$ can be naturally identified with the space $\mathcal{H}_{a}$.

Our third main result gives the relationship between the operator $A_{1}$ and the generator of the Lax-Phillips semigroup. 
Theorem 2.3. The generator $\mathcal{B}$ of the Lax-Phillips semigroup $Z_{t}$ coincides with the operator $A_{1}$ defined by the differential expression (1.11) on the domain $D\left(A_{1}\right)$ given in (1.16) with $h=1$.

To prove this theorem we obtain one more result, which might be of interest in itself. Namely, we obtain an explicit formula for the resolvent of the operator $\mathcal{B}$. This formula is the content of Theorem 4.2, which is formulated and proven in Section 4 .

Remark 2.1. We define resonances as the eigenvalues of the operator $A_{1}$. This definition is used as a standard definition of resonances in the study of the so-called Regge problem [12]. We recall that the Regge problem consists in the solving of the nonstandard boundary-value problem (4.1) and (4.2) (see Section 4). At the same time, the notion of resonances is also used in the scattering theory. We recall that in the Lax-Phillips scattering theory resonances are defined as eigenvalues of the generator $\mathcal{B}$ of the Lax-Phillips semigroup. Based on Theorem 2.3, we see that the problem considered in the present paper provides an exclusive example when the generator of the Lax-Phillips semigroup admits an explicit description in the form of a matrix differential operator with special nonselfadjoint boundary conditions.

\section{Properties of Operators $A_{h}$. Proof of Theorems 2.1 and 2.2}

In this section, we prove the results about the properties of the operator $A_{h}$. As it will follow from the proofs below, $A_{h}$ can fail to be dissipative if we allow the parameter $h$ to be an arbitrary complex number. But the other two properties of $A_{h}$ (namely, maximality and simplicity) will survive. As in applications (e.g., the theory of semigroups, control theory, etc.), the operator $A_{h}$ appears as a generator of certain semigroup, it is of a particular importance to discuss the cases when $A_{h}$ is a dissipative operator (and the related semigroup is a semigroup of contractions). That is why we keep the requirement $R e h \geq 0$, which leads to the disspativity of $A_{h}$.

Proof of Theorem 2.1. First of all, we prove that $A_{h}$ with $R e h \geq 0$ is dissipative in $\mathcal{H}_{a}$. By a straightforward calculation, we have for any $U \in D\left(A_{h}\right)$

$$
\begin{aligned}
\left(A_{h} U, U\right)_{\mathcal{H}_{a}}= & -\frac{i}{2} p(a) u_{0}^{\prime}(a) \overline{u_{1}(a)}+i \int_{0}^{a} d(x) \rho(x)\left|u_{1}(x)\right|^{2} d x \\
& +\operatorname{Im} \int_{0}^{a}\left[\bar{u}_{0}^{\prime}(x) u_{1}^{\prime}(x) p(x)+\bar{u}_{0}(x) u_{1}(x) q(x) \rho(x)\right] d x .
\end{aligned}
$$

From the boundary conditions (1.16), it follows that

$$
u_{0}^{\prime}(a)=-h u_{1}(a)
$$

and, therefore,

$$
\operatorname{Im}\left(A_{h} U, U\right)_{\mathcal{H}_{a}}=\frac{1}{2} \operatorname{Re} h\left|u_{1}(a)\right|^{2} p(a)+\left\|\sqrt{d \rho} u_{1}\right\|_{L^{2}(0, a)}^{2} \geq 0 .
$$

(3.1) means that $A_{h}$ is dissipative.

Now let us prove that $A_{h}$ is simple. To do this we have to show that both operators $A_{h}$ and $A_{h}^{*}$ do not have mutual invariant subspaces where they coincide. 
First of all we derive the formula for the adjoint operator. For any $U \in D\left(A_{h}\right)$

$$
\begin{aligned}
& \left(A_{h} U, V\right)_{\mathcal{H}_{a}}=-\frac{i}{2}\left[p(0) u_{0}^{\prime}(0) \overline{v_{1}(0)}-p(a) u_{1}(a) \overline{v_{0}^{\prime}(a)}-p(a) u_{0}^{\prime}(a) \overline{v_{1}(a)}\right] \\
& +\frac{i}{2} \int_{0}^{a}\left[u_{1}(x)\left(p(x) \bar{v}_{0}^{\prime}\right)^{\prime}+u_{0}^{\prime}\left(p(x) \bar{v}_{1}^{\prime}\right)^{\prime}+2 d(x) \rho(x) u_{0} \bar{v}_{1}+q(x) \rho(x)\left(u_{0} \bar{v}_{1}-u_{1} \bar{v}_{0}\right)\right] d x .
\end{aligned}
$$

Due to the boundary conditions (1.16)

$$
u_{1}(a) \overline{v_{0}^{\prime}(a)}+u_{0}^{\prime}(a) \overline{v_{1}(a)}=u_{1}(a) \overline{\left[v_{0}^{\prime}(a)-h v_{1}(a)\right]} .
$$

Based on the above two relations, we obtain the following formula for the adjoint operator:

$$
\begin{gathered}
A_{h}^{*}=-i\left(\begin{array}{cc}
0 & 1 \\
\frac{1}{\rho(x)} \frac{d}{d x}\left(p(x) \frac{d}{d x} \cdot\right)-q(x) . & 2 d(x)
\end{array}\right), \\
D\left(A_{h}^{*}\right)=\left\{V=\left(\begin{array}{c}
v_{0} \\
v_{1}
\end{array}\right) \in \mathcal{H}_{a}: v_{0}^{\prime \prime}, v_{0}^{\prime} \in \mathbb{L}^{2}(0, a),\right. \\
\left.v_{1}(0)=0, \quad v_{0}^{\prime}(a)-\bar{h} v_{1}(a)=0\right\} .
\end{gathered}
$$

We will prove that $A_{h}$ is simple using a contradiction argument. Assume that there exists a nonzero vector $F \in \mathcal{H}_{a}$ such that

$$
F=\left(\begin{array}{c}
f_{0} \\
f_{1}
\end{array}\right) \in D\left(A_{h}\right) \cap D\left(A_{h}^{*}\right) \text { and } A_{h} F=A_{h}^{*} F
$$

Rewriting the equation from (3.4) in component-wise form, we easily obtain that $d(x) f_{1}(x)=0$. Since $d(x)>0$ (see $(1.4)$ ), we have $f_{1}(x)=0$. The latter fact implies that the vectors from the mutual invariant subspace must be of the form $F=\left(\begin{array}{c}f_{0} \\ 0\end{array}\right)$, and, therefore, $A_{h} F$ must be of the same form. But

$$
A_{h}\left(\begin{array}{c}
f_{0} \\
0
\end{array}\right)=\left(\begin{array}{c}
0 \\
\frac{1}{\rho(x)} \frac{d}{d x}\left(p(x) \frac{d f_{0}}{d x}\right)-q(x) f_{0}(x)
\end{array}\right) .
$$

(3.5) means that $f_{0}$ must satisfy the following homogeneous equation:

$$
\left(p(x) f_{0}^{\prime}(x)\right)^{\prime}-q(x) \rho(x) f_{0}(x)=0 .
$$

From Eq. (3.6), we derive that there must exist an eigenvector of $A_{h}$ (as well as of $A_{h}^{*}$ ) corresponding to the zero eigenvalue. Let us show that this is impossible. Multiplying Eq. (3.6) by $\bar{f}_{0}(x)$ and integrating, we obtain

$$
p(a) f_{0}^{\prime}(a) \overline{f_{0}(a)}-p(0) f_{0}^{\prime}(0) \overline{f_{0}(0)}-\int_{0}^{a} p(x)\left|f_{0}^{\prime}\right|^{2} d x=\int_{0}^{a} q(x) \rho(x)\left|f_{0}\right|^{2} d x .
$$

From boundary conditions (1.16) we have $f_{0}(a)=0$, and due to the definition of the space $\mathcal{H}_{a}$ we have $f_{0}(0)=0$. It is clear that each integral in Eq. (3.7) must be equal to zero, which is possible only if $f_{0}=0$. The latter means that $A_{h}$ is simple. 
To complete the proof of the theorem, we have to show that $A_{h}$ is maximal, which means that $A_{h}$ does not admit any extensions. So, it suffices to prove (see, e.g., $[4,5])$ that

$$
\overline{\left(A_{h}+i I\right) D\left(A_{h}\right)}=\mathcal{H}_{a},
$$

where the bar denotes closure. Eq. (3.8) is equivalent to the fact that the equation

$$
\left(A_{h}^{*}-i I\right) F=0
$$

has only the trivial solution. Let us prove this. For any $F=\left(\begin{array}{c}f_{0} \\ f_{1}\end{array}\right)$, Eq. (3.9) is equivalent to the following system:

$$
\begin{gathered}
f_{1}=-f_{0}, \\
\frac{1}{\rho(x)}\left(p(x) f_{0}^{\prime}\right)^{\prime}-q(x) f_{0}+2 d(x) f_{1}=-f_{1} .
\end{gathered}
$$

From (3.10) and (3.11) we derive the following equation for $f_{0}$ :

$$
\frac{1}{\rho(x)}\left(p(x) f_{0}^{\prime}\right)^{\prime}-q(x) f_{0}(x)+2 d(x) f_{0}(x)=f_{0}(x)
$$

with the boundary conditions

$$
f_{0}(0)=0, \quad f_{0}^{\prime}(a)+\bar{h} f_{0}(a)=0, \quad R e h \geq 0 .
$$

The problem defined by (3.12), (3.13) may have only the trivial solution. Indeed, multiplying (3.12) by $\bar{f}_{0}$ and integrating, we have

$$
\int_{0}^{a}\left(p(x) f_{0}^{\prime}\right)^{\prime} \bar{f}_{0} d x-\int_{0}^{a}(q(x)+2 d(x) \rho(x))\left|f_{0}\right|^{2} d x=\int_{0}^{a} \rho(x)\left|f_{0}\right|^{2} d x .
$$

Eq. (3.14) can be transformed to

$$
p(a) f_{0}^{\prime}(a) \overline{f_{0}(a)}=\left\|f_{0}^{\prime}\right\|_{L^{2}(0, a)}^{2}+\left\|\sqrt{\rho(q+2 d)} f_{0}\right\|_{L^{2}(0, a)}^{2}+\left\|\sqrt{\rho} f_{0}\right\|_{L^{2}(0, a)}^{2} .
$$

If $f_{0}^{\prime}(a)$ or $f_{0}(a)$ vanishes, $(3.15)$ is impossible for a nontrivial function $f_{0}$. Let us consider the case when neither $f_{0}(a)$ nor $f_{0}^{\prime}(a)$ is equal to zero.

Due to boundary conditions (3.13), we have

$$
p(a) f_{0}^{\prime}(a) \overline{f_{0}(a)}=-h\left|f_{0}(a)\right|^{2} .
$$

Based on (3.16), we can see that the left-hand side of Eq. (3.15) has nonpositive real part while its right-hand side is always positive unless $f_{0}=0$. This contradiction proves the maximality. Theorem 2.1 is proved.

Remark 3.1. Notice that if we consider the operator $\mathfrak{L}_{0}^{*}$ adjoint to $\mathfrak{L}_{0}$ defined by (2.1), then $\mathfrak{L}_{0}^{*}$ is defined by the same differential expression (3.2) with the Dirichlet boundary condition at $x=0$ and without any boundary condition at $x=a$, i.e.,

$$
D\left(\mathfrak{L}_{0}^{*}\right)=\left\{V=\left(\begin{array}{c}
v_{0} \\
v_{1}
\end{array}\right) \in \mathcal{H}_{a}: v_{0}^{\prime \prime}, v_{1}^{\prime} \in \mathbb{L}^{2}(0, a) v_{1}(0)=0\right\} .
$$

Let us study the equation similar to $(3.10)$ for $\mathfrak{L}_{0}^{*}$ :

$$
\left(\mathfrak{L}_{0}^{*}-i I\right) F=0 .
$$

Completing all steps similar to (3.10) - (3.14), we arrive at Eq. (3.15). But this time due to the fact that $F \in D\left(\mathfrak{L}_{0}^{*}\right)$ there are no restrictions on the behavior of 
the function $f_{0}(x)$ at $x=a$. This fact means that Eq. (3.18) may have nontrivial solutions. The next theorem answers the question about the number of linearly independent solutions of this equation.

Proof of Theorem 2.2. First of all let us show that the domain of any operator $A_{h}$ is represented as the following direct (but not necessarily orthogonal) sum:

$$
D\left(A_{h}\right)=D\left(\mathfrak{L}_{0}\right) \oplus \mathfrak{V}_{h},
$$

where $\mathfrak{V}_{h}$ is a one dimensional subspace. We give an explicit formula for this subspace. Namely, we prove that this subspace is spanned by the vector $X$ given by

$$
X(x)=\left(\begin{array}{c}
x \\
-x / a h
\end{array}\right) \text { if } h \neq 0, \quad X(x)=\left(\begin{array}{c}
\frac{1}{2 a} x^{2}-x \\
0
\end{array}\right) \text { if } h=0, \quad x \in[0, a] .
$$

We prove (3.19) only for $h \neq 0$. For $h=0$, the proof is similar.

The proof of (3.19) splits into two steps. We have to establish two facts:

a) For any $V=\left(\begin{array}{l}v_{0} \\ v_{1}\end{array}\right) \in D\left(A_{h}\right)$ there exists a constant $C$ such that $\widetilde{V}=V-$ $C X \in D\left(\mathfrak{L}_{0}\right)$. The latter means

$$
D\left(A_{h}\right) \subseteq D\left(\mathfrak{L}_{0}\right) \oplus \mathfrak{V}_{h} .
$$

b) For any $\widetilde{V}=\left(\begin{array}{l}\widetilde{v}_{0} \\ \widetilde{v}_{1}\end{array}\right) \in D\left(\mathfrak{L}_{0}\right)$ a linear combination $V=\widetilde{V}+C X, C \in \mathbb{C}$ belongs to $D\left(A_{h}\right)$. The latter means

$$
D\left(\mathfrak{L}_{0}\right) \oplus \mathfrak{V}_{h} \subseteq D\left(A_{h}\right) .
$$

To prove statement a), we notice that for any $V \in D\left(A_{h}\right)$ the combination $\widetilde{V}=V-C_{0} X$ with $C_{0}=v_{0}^{\prime}(a)$ belongs to $D\left(\mathfrak{L}_{0}\right)$. Indeed,

$$
\widetilde{V}(x)=\left(\begin{array}{l}
\widetilde{v}_{0}(x) \\
\widetilde{v}_{1}(x)
\end{array}\right)=\left(\begin{array}{l}
v_{0}(x)-v_{0}^{\prime}(a) x \\
v_{1}(x)+v_{0}^{\prime}(a) x / a h
\end{array}\right) .
$$

Now we verify the boundary conditions $(2.1)$. We have

$$
\begin{gathered}
\widetilde{v}_{1}(0)=v_{1}(0)=0, \\
v_{0}^{\prime}(a)=\left(v_{0}-v_{0}^{\prime}(a) x\right)^{\prime}=0, \quad \widetilde{v}_{1}(a)=v_{1}(a)+v_{0}^{\prime}(a) h^{-1}=0 .
\end{gathered}
$$

In the last step, we used the fact that $V \in D\left(A_{h}\right)$ and that the components $v_{1}$ and $v_{0}^{\prime}$ satisfy (1.16). Therefore, inclusion (3.21) is shown. Inclusion (3.22) is obvious because $X \in D\left(A_{h}\right)$ and $D\left(\mathfrak{L}_{0}\right) \subset D\left(A_{h}\right)$. Thus, Eq. (3.19) is proved.

Now we prove that for any $h \in \mathbb{C}$, the operator $A_{h}$ is a one-dimensional extension of $\mathfrak{L}_{0}$. First of all, we note that the whole lower half-plane of $\lambda$ is the resolvent set for both operators $A_{h}$ and $\mathfrak{L}_{0}$. Therefore, to prove Theorem 2.2 , it suffices to show that the difference of the corresponding resolvents $\mathcal{R}_{\lambda}\left(A_{h}\right)$ and $\mathcal{R}_{\lambda}\left(\mathfrak{L}_{0}\right)$ is a one-dimensional operator [2]. Moreover, this difference can be evaluated at any point $\lambda$ with $\operatorname{Im} \lambda<0$, for example, $\lambda=-i$. Let us denote by $\mathcal{N}\left(\mathfrak{L}_{0}^{*}-i I\right)$ the null-space of the operator $\mathcal{L}_{0}^{*}-i I$. We have

$$
\left(A_{h}+i I\right)^{-1} \mathcal{H}_{a}=D\left(\mathfrak{L}_{0}\right) \oplus\left(A_{h}+i I\right)^{-1} \mathcal{N}\left(\mathfrak{L}_{0}^{*}-i I\right),
$$




$$
\left(\mathfrak{L}_{0}+i I\right)^{-1} \mathcal{H}_{a}=D\left(\mathfrak{L}_{0}\right) \oplus\left(\mathfrak{L}_{0}+i I\right)^{-1} \mathcal{N}\left(\mathfrak{L}_{0}^{*}-i I\right),
$$

where by $\oplus$ we denote the direct (but not necessarily orthogonal) sum of subspaces. (3.24) and (3.25) follow from the standard orthogonal decomposition

$$
\mathcal{H}_{a}=R\left(\mathfrak{L}_{0}+i I\right) \oplus \mathcal{N}\left(\mathfrak{L}_{0}^{*}-i I\right),
$$

where

$$
R\left(\mathfrak{L}_{0}+i I\right)=\left(\mathfrak{L}_{0}+i I\right) D\left(\mathfrak{L}_{0}\right)=\left(A_{h}+i I\right) D\left(\mathfrak{L}_{0}\right) .
$$

It is shown in our paper [20] that the equation

$$
\left(\mathfrak{L}_{0}^{*}-i I\right) \Phi=0
$$

has only one solution $\Phi \in D\left(\mathfrak{L}_{0}^{*}\right)$ (see (3.17)). Thus, the null-space $\mathcal{N}\left(\mathfrak{L}_{0}^{*}-i I\right)$ is one-dimensional and is spanned by the vector $\left(\begin{array}{r}\varphi(x) \\ -\varphi(x)\end{array}\right)$, where $\varphi(x)$ is a solution of the following problem:

$$
u^{\prime \prime}-\rho(x)(1+2 d(x)) u=0, \quad u(0)=0 .
$$

From (3.24) and (3.25) we obtain

$$
\left[\mathcal{R}_{-i}\left(A_{h}\right)-\mathcal{R}_{-i}\left(\mathfrak{L}_{0}\right)\right] \mathcal{H}_{a}=\left[\mathcal{R}_{-i}\left(A_{h}\right)-\mathcal{R}_{-i}\left(\mathfrak{L}_{0}\right)\right] \mathcal{N}\left(\mathfrak{L}_{0}^{*}-i I\right) .
$$

Now the desired result follows from the fact that $\operatorname{dim} \mathcal{N}\left(\mathfrak{L}_{0}^{*}-i I\right)=1$. Theorem 2.2 is proved.

Proof of Corollary 2.1. Because (3.24) is valid for any $h$, we can write the following relation for any $h_{1}$ and $h_{2}$ :

$$
\left[\mathcal{R}_{-i}\left(A_{h_{1}}\right)-\mathcal{R}_{-i}\left(A_{h_{2}}\right)\right] \mathcal{H}_{a}=\left[\mathcal{R}_{-i}\left(A_{h_{1}}\right)-\mathcal{R}_{-i}\left(A_{h_{2}}\right)\right] \mathcal{N}\left(\mathfrak{L}_{0}^{*}-i I\right),
$$

which proves this corollary.

Remark 3.2. As was explained in the Introduction, in what follows one of the operators from the set $A_{h}$ is of particular importance for us. It corresponds to $h=1$ and is denoted by $A_{1}$. The importance of $A_{1}$ is related to the fact that $A_{1}$ appears in the Lax-Phillips scattering theory as a generator of the semigroup $Z_{t}$. So, to study this operator, we can invoke the so-called incoming (or outgoing) translation representation from the Lax-Phillips approach and formulate the whole problem in the language of the functional model for nonselfadjoint operators. Reduction to the functional model allows us to clarify the problem related to such properties of the set of eigenfunctions of $A_{1}$ in the space $\mathcal{H}_{a}$ as completeness, minimality, uniform minimality and even the basis property.

\section{Generator of Lax-Phillips Semigroup}

In this section, we prove our third main result - Theorem 2.3. This proof is based on detailed information about the eigenfunctions and eigenvalues of the family of nonselfadjoint quadratic operator pencils naturally related to the operators $A_{h}$ $[19,20]$.

To simplify the exposition we represent some necessary statements from $[19,20]$ in the form of Theorem 4.1 below. 
First we define the aforementioned pencils. Note that the eigenvector equation

$$
A_{h} U=\lambda U, \quad U(x)=\left(\begin{array}{c}
u_{0}(x) \\
u_{1}(x)
\end{array}\right),
$$

is equivalent to the following boundary problem for the function $u(x)=u_{0}(x)$ :

$$
\begin{aligned}
& \frac{1}{\rho(x)} \frac{d}{d x}\left(p(x) \frac{d u(x)}{d x}\right)+\lambda^{2} u(x)-2 i \lambda d(x) u(x)-q(x) u(x)=0, \\
& u(0)=0, \quad\left(u_{x}+i \lambda h u\right)(a)=0 .
\end{aligned}
$$

Problem (4.1), (4.2) defines a one parameter family (with parameter $h$ ) of nonselfadjoint quadratic operator pencils. The eigenvalues of the pencil (for fixed $h$ ) coincide with the eigenvalues of $A_{h}$. The eigenvectors of the pencil are exactly the first component eigenvectors of $A_{h}$.

Theorem 4.1. a) For any $\lambda \in \mathbb{C}$ there exists a unique solution $J(\lambda, x)$ of Eq. (4.1) which satisfies the condition at $x=$ a from (4.2) with $h=1$ and the normalization condition: $J(\lambda, a)=1$. $J(\lambda, x)$ is called the Jost solution $([12])$.

b) We call the function

$$
\mathbf{J}(\lambda)=J(\lambda, 0)
$$

the Jost function [12]. $\mathbf{J}(\lambda)$ is an entire function of the first order and of exponential type $\mathcal{M}$ with $\mathcal{M}$ defined in (1.7).

c) The Jost function has an infinite set of roots. All roots, except for, maybe, a finite number of them are simple. All roots are located in a strip parallel to the real axis and the set of roots is symmetric with respect to the imaginary axis; multiplicities of any two symmetric roots are equal. Only a finite number of roots may be purely imaginary. The set of roots has only two points of accumulation: $+\infty$ and $-\infty$.

d) There exists a unique solution $\Phi(\lambda, x)$ of $E q$. (4.1) defined on the semi-axis $x \in[0, \infty)$, satisfying the conditions

$$
\Phi(\lambda, 0)=0, \quad \Phi_{x}(\lambda, 0)=1 .
$$

If the Jost solution is extended for $x \in(a, \infty)$ as

$$
J(\lambda, x)=e^{-i \lambda x}, \quad x \geq a,
$$

then the Wronskian of these two solutions can be given by the formula

$$
\{J(\lambda, x), \Phi(\lambda, x)\}=\mathbf{J}(\lambda) .
$$

In the future the solution $\Phi(\lambda, x)$ with $\lambda \in \mathbb{R}$ will be called an eigenfunction of a continuous spectrum of the following quadratic operator pencil in the weighted space $\mathbb{L}^{2}(0, \infty)$ (the norm in this space is given by (1.6) with " $a$ " replaced by $\infty$ ):

$$
L_{\lambda}=L_{0}-\lambda^{2} I+2 i \lambda d(x), \quad x \in[0, \infty) .
$$

Here $L_{0}$ is the selfadjoint operator in $\mathbb{L}^{2}(0, \infty)$ defined by

$$
\left(L_{0} u\right)(x)=-\frac{1}{\rho(x)} \frac{d}{d x}\left(p(x) \frac{d u(x)}{d x}\right)+q(x) u(x)
$$

on the domain

$$
D\left(L_{0}\right)=\left\{u \in \mathbb{L}^{2}(0, \infty): u^{\prime}, u^{\prime \prime} \in \mathbb{L}^{2}(0, \infty), u(0)=0\right\} .
$$


e) For any $f \in \mathbb{L}^{2}(0, \infty)$ the equation $L_{\lambda} g=f$, Im $\lambda<0$, has a unique solution denoted by $L_{\lambda}^{-1} f$ that can be represented as

$$
\begin{aligned}
\left(L_{\lambda}^{-1} f\right)(x)= & {[\mathbf{J}(\lambda)]^{-1}\left[J(\lambda, x) \int_{0}^{x} \Phi(\lambda, \xi) \rho(\xi) p(\xi)^{-1} f(\xi) d \xi\right.} \\
& \left.+\Phi(\lambda, x) \int_{x}^{\infty} J(\lambda, \xi) \rho(\xi) p(\xi)^{-1} f(\xi) d \xi\right]
\end{aligned}
$$

Now we are in position to give our first main result of this section.

Theorem 4.2. The resolvent $\mathcal{R}_{\lambda}(\mathcal{B})$ of the generator of the semigroup $Z_{t}(2.5)$ can be given in the following form:

$$
\mathcal{R}_{\lambda}(\mathcal{B})=\left(\begin{array}{cr}
\mathbb{L}_{\lambda} & -i L_{\lambda}^{-1} \\
i \lambda \mathbb{L}_{\lambda}+i I & \lambda L_{\lambda}^{-1}
\end{array}\right)
$$

where

$$
\mathbb{L}_{\lambda}=L_{\lambda}^{-1}(\lambda-2 i d) \mathcal{X}_{a}-i \mathbf{J}(\lambda)^{-1} \Phi(\lambda, \cdot) e^{-i \lambda a} P_{a}
$$

$\mathcal{X}_{a}$ - is the characteristic function of the interval $[0, a], \Phi(\lambda, x)$ is the above described eigenfunction of the continuous spectrum of the quadratic operator pencil (4.6) (4.8), and $P_{a}$ is the functional defined on continuous functions by the formula $\left(P_{a} g\right)(x)=g(a)$.

Remark 4.1. We notice that the operator given by (4.10) is well defined on the whole energy space $\mathcal{H}$. Indeed, if this operator is applied to any vector $\left(\begin{array}{c}u_{0} \\ u_{1}\end{array}\right) \in \mathcal{H}$, then $\mathbb{L}_{\lambda}$ acts only on $u_{0}$. But the weak derivative $u_{0}^{\prime} \in L^{2}(0, \infty)$ and, therefore, $u_{0}$ must be absolutely continuous. So, $P_{a} u_{0}=u_{0}(a)$ makes sense.

Proof of Theorem 4.2. Let us return to the semigroup $\left\{V_{t}\right\}_{t \leq 0}$. The generator of this semigroup, denoted by $\mathfrak{L}$, is the differential operator in $\mathcal{H}$ given by the matrix differential expression (1.11) on the domain

$$
D(\mathfrak{L})=\left\{U=\left(\begin{array}{l}
u_{0} \\
u_{1}
\end{array}\right) \in \mathcal{H}: u_{0}^{\prime \prime}, u_{1}^{\prime} \in \mathbb{L}^{2}(0, \infty), u_{1} \in H^{1}(0, \infty), u_{1}(0)=0\right\} .
$$

Let $\mathcal{R}_{\lambda}(\mathfrak{L})$ be the resolvent operator for $\operatorname{Im} \lambda<0$. Then the following relation holds for the resolvent operator $\mathcal{R}_{\lambda}(\mathcal{B})$ of the generator $\mathcal{B}$ of the semigroup $Z_{t}([8])$ :

$$
\mathcal{R}_{\lambda}(\mathcal{B})=\left.P_{K} \mathcal{R}_{\lambda}(\mathfrak{L})\right|_{K}, \quad \operatorname{Im} \lambda<0
$$

Therefore, to prove the formula for $\mathcal{R}_{\lambda}(\mathcal{B})$ we need the formula for $\mathcal{R}_{\lambda}(\mathfrak{L})$. To derive this formula, let us consider the following nonhomogeneous differential equation:

$$
\mathfrak{L} G-\lambda G=F, \quad \operatorname{Im} \lambda<0
$$

in the energy space $\mathcal{H}$. In Eq. (4.14), we use the notation $G=\left(\begin{array}{c}g_{0} \\ g_{1}\end{array}\right) \in D(\mathfrak{L})$, and $F=\left(\begin{array}{c}f_{0} \\ f_{1}\end{array}\right)$ is an arbitrary vector from $\mathcal{H}$. Eq. (4.14) is equivalent to the 
system

$$
\begin{gathered}
-\lambda g_{0}-i g_{1}=f_{0}, \\
i L_{0} g_{0}+(2 i d-\lambda) g_{1}=f_{1},
\end{gathered}
$$

where $L_{0}$ is defined by (4.7), (4.8). System (4.15), (4.16) can be reduced to the following equation for $g_{0}$ :

$$
\left(L_{0}-\lambda^{2}+2 i \lambda d\right) g_{0}=-i f_{1}+(\lambda-2 i d) f_{0} .
$$

Based on statement e) of Theorem 4.1 we obtain from (4.15) - (4.17) that the following formula holds for the solution $G$ of Eq. (4.14):

$$
G=\left(\begin{array}{c}
g_{0} \\
g_{1}
\end{array}\right)=\left(\begin{array}{c}
L_{\lambda}^{-1}\left[(\lambda-2 i d) f_{0}\right]-i L_{\lambda}^{-1} f_{0} \\
i\left[\lambda L_{\lambda}^{-1}(\lambda-2 i d)+I\right] f_{0}+\lambda L_{\lambda}^{-1} f_{1}
\end{array}\right) .
$$

From (4.18) we obtain the formula for the resolvent:

$$
\mathcal{R}_{\lambda}(\mathfrak{L})=\left(\begin{array}{ll}
L_{\lambda}^{-1}(\lambda-2 i d) & -i L_{\lambda}^{-1} \\
i\left[\lambda L_{\lambda}^{-1}(\lambda-2 i d)+I\right] & \lambda L_{\lambda}^{-1}
\end{array}\right) .
$$

Notice that when $d=0$, we obtain the result established in our older paper [14]:

$$
\mathcal{R}_{\lambda}(\mathfrak{L})=\left(\begin{array}{ll}
\lambda R_{\lambda^{2}}\left(L_{0}\right) & -i R_{\lambda^{2}}\left(L_{0}\right) \\
i \lambda^{2} R_{\lambda^{2}}\left(L_{0}\right)+i I & \lambda R_{\lambda^{2}}\left(L_{0}\right)
\end{array}\right),
$$

where $R_{\lambda^{2}}\left(L_{0}\right)$ is the resolvent of the operator $L_{0}$ defined by (4.7), (4.8).

Now let us derive the formula for the restriction $\left.\mathcal{R}_{\lambda}(\mathfrak{L})\right|_{K}$ of the operator $\mathcal{R}_{\lambda}(\mathfrak{L})$ to the subspace $K$. By Lemma 2.1, if $\widetilde{G} \in \mathcal{H}$, then $G=P_{K} \widetilde{G}=\left(\begin{array}{c}g_{0} \\ g_{1}\end{array}\right) \in K$ if and only if

$$
\begin{array}{ll}
g_{0}(x)=\widetilde{g}_{0}(x) \text { for } x \leq a & \text { and } g_{0}(x)=\widetilde{g}_{0}(a) \text { for } x>a \\
g_{1}(x)=\widetilde{g}_{1}(x) \text { for } x \leq a & \text { and } g_{1}(x)=0 \text { for } x>a .
\end{array}
$$

Using (4.11), (4.19) and (4.20) we obtain that

$$
\mathcal{R}_{\lambda}(\mathfrak{L}) G=\widetilde{F} \equiv\left(\begin{array}{c}
\tilde{f}_{0} \\
\tilde{f}_{1}
\end{array}\right),
$$

with the following expressions for both components:

$$
\widetilde{f}_{0}=L_{\lambda}^{-1}\left((\lambda-2 i d) g_{0}-i g_{1}\right),
$$

or

$$
\begin{gathered}
\widetilde{f}_{0}(x)=[\mathbf{J}(\lambda)]^{-1}\left\{J(\lambda, x) \int_{0}^{x} \Phi(\lambda, \xi) \rho(\xi) p(\xi)^{-1}\left[(\lambda-2 i d(\xi)) g_{0}(\xi)-i g_{1}(\xi)\right] d \xi\right. \\
\left.+\Phi(\lambda, x) \int_{x}^{\infty} J(\lambda, \xi) \rho(\xi) p(\xi)^{-1}\left[(\lambda-2 i d(\xi)) g_{0}(\xi)-i g_{1}(\xi)\right] d \xi\right\} \\
\tilde{f}_{1}(x)=i \lambda \widetilde{f}_{0}(x)+i g_{0}(x) .
\end{gathered}
$$


Our final step is to give a description of the projection of the vector $\widetilde{F}$ on the subspace $K$.

Using the facts that $J(\lambda, x)=e^{-i \lambda x}, g_{0}(x)=g_{0}(a), g_{1}(x)=0$ for $x \in(a, \infty)$ and $\operatorname{Im} \lambda<0$, we can rewrite (4.21), (4.22) for $x \in[0, a]$ in the forms

$$
\begin{aligned}
\tilde{f}_{0}(x)= & \frac{1}{\mathbf{J}(\lambda)}\left\{J(\lambda, x) \int_{0}^{x} \Phi(\lambda, \xi) \rho(\xi) p(\xi)^{-1}\left((\lambda-2 i d(\xi)) g_{0}(\xi)-i g_{1}(\xi)\right) d \xi\right. \\
& +\Phi(\lambda, x) \int_{x}^{a} J(\lambda, \xi) \rho(\xi) p(\xi)^{-1}\left((\lambda-2 i d(\xi)) g_{0}(\xi)-i g_{1}(\xi)\right) d \xi \\
& \left.-i \Phi(\lambda, x) e^{-i \lambda a} g_{0}(a)\right\}
\end{aligned}
$$

$$
\begin{aligned}
\widetilde{f}_{1}(x)= & \frac{i \lambda}{\mathbf{J}(\lambda)}\left\{J(\lambda, x) \int_{0}^{x} \Phi(\lambda, \xi) \rho(\xi) p(\xi)^{-1}((\lambda-2 i d \xi)) g_{0}(\xi)-i g_{1}(\xi)\right) d \xi \\
& \left.-\Phi(\lambda, x) \int_{x}^{a} J(\lambda, \xi) \rho(\xi) p(\xi)^{-1}\left((\lambda-2 i d(\xi)) g_{0}(\xi)-i g_{1}(\xi)\right) d \xi\right\} \\
& +\frac{\lambda}{\mathbf{J}(\lambda)} g_{0}(a) e^{-i \lambda a} \Phi(\lambda, x)+i g_{0}(x) .
\end{aligned}
$$

We can represent (4.23) and (4.24) in the forms

$$
\begin{aligned}
& \widetilde{f}_{0}=L_{\lambda}^{-1}\left(\left[\lambda g_{0}-2 i d g_{0}-i g_{1}\right] \mathcal{X}_{a}\right)-i[\mathbf{J}(\lambda)]^{-1} \Phi(\lambda, \cdot) e^{-i \lambda a} g_{0}(a), \\
& \widetilde{f}_{1}=i \lambda L_{\lambda}^{-1}\left(\left[\lambda g_{0}-2 i d g_{0}-i g_{1}\right] \mathcal{X}_{a}\right)+\lambda[\mathbf{J}(\lambda)]^{-1} \Phi(\lambda, \cdot) e^{-i \lambda a} g_{0}(a)+i g_{0} .
\end{aligned}
$$

Collecting together (4.25), (4.26) and using Lemma 2.1, we obtain the following formulas: for $x \in[0, a]$,

$$
=\left(\begin{array}{l}
\left(L_{\lambda}^{-1}\left[g_{0}(\lambda-2 i d)-i g_{1}\right] \mathcal{X}_{a}\right)(x)-i[\mathbf{J}(\lambda)]^{-1} \Phi(\lambda, x) e^{-i \lambda a} g_{0}(a) \\
i \lambda\left(L_{\lambda}^{-1}\left[g_{0}(\lambda-2 i d)-i g_{1}\right] \mathcal{X}_{a}\right)(x)+2[\mathbf{J}(\lambda)]^{-1} \Phi(\lambda, x) e^{-i \lambda a} g_{0}(a)+i g_{0}(x)
\end{array}\right),
$$

for $x>a$,

$$
\begin{aligned}
& \left(\mathcal{R}_{\lambda}(\mathcal{B}) G\right)(x) \\
& =\left(\begin{array}{c}
\left(L_{\lambda}^{-1}\left[g_{0}(\lambda-2 i d)-i g_{1}\right] \mathcal{X}_{a}\right)(a)-i[\mathbf{J}(\lambda)]^{-1} \Phi(\lambda, a) e^{-i \lambda a} g_{0}(a) \\
0
\end{array}\right) .
\end{aligned}
$$

Expression (4.10) follows immediately from (4.27) and (4.28). This proves Theorem 4.2 .

Now we are in a position to prove Theorem 2.3.

Proof of Theorem 2.3. To prove that $\mathcal{B}=A_{1}$ it is sufficient to show (see $\left.[2,7]\right)$ that

$$
\mathcal{R}_{\lambda}(\mathcal{B})=\mathcal{R}_{\lambda}\left(A_{1}\right), \quad \operatorname{Im} \lambda<0 .
$$


To do this, we have to demonstrate that for any vector $G=\left(\begin{array}{c}g_{0} \\ g_{1}\end{array}\right) \in \mathcal{H}_{a}$ the following two facts hold:
a) $\widetilde{F}=\left(\begin{array}{c}\tilde{f}_{0} \\ \widetilde{f}_{1}\end{array}\right)=\mathcal{R}_{\lambda}(\mathcal{B})\left(\begin{array}{c}g_{0} \\ g_{1}\end{array}\right) \in D\left(A_{1}\right)$,
b) $\left(A_{1}-\lambda I\right) \widetilde{F}=\left(A_{1}-\lambda I\right) \mathcal{R}_{\lambda}(\mathcal{B}) G=G$.

To check a) it suffices to show that $\left(\begin{array}{c}\tilde{f}_{0} \\ \widetilde{f}_{1}\end{array}\right)$ satisfies the boundary conditions $\widetilde{f}_{1}(0)=0$ and $\left(\widetilde{f}_{0}^{\prime}+\widetilde{f}_{1}\right)(a)=0$. Evaluating $(4.25)$ at $x=0$, we obtain

$$
\begin{aligned}
\widetilde{f}_{1}(0)= & \frac{\Phi(\lambda, 0)}{\mathbf{J}(\lambda)} \int_{0}^{a} J(\lambda, \xi) \rho(\xi) p(\xi)^{-1}\left((\lambda-2 i d(\xi)) g_{0}(\xi)-i g_{1}(x)\right) d \xi \\
& +\frac{\lambda}{\mathbf{J}(\lambda)} \Phi(\lambda, 0) g_{0}(a) e^{-i \lambda a}+i g_{0}(0)
\end{aligned}
$$

where we have taken into account that $g_{0}(0)=0$, due to the definition of the energy space, and $\Phi(\lambda, 0)=0$ due to (4.4).

Now we verify the second boundary condition. From (4.25) we have

$$
\begin{aligned}
\widetilde{f}_{1}(a)= & \frac{i \lambda}{\mathbf{J}(\lambda)} J(\lambda, a) \int_{0}^{a} \Phi(\lambda, \xi) \rho(\xi) p(\xi)^{-1}\left(\left(\lambda-2 i d(\xi) g_{0}(\xi)-i g_{1}(\xi)\right) d \xi\right. \\
& +\frac{\lambda}{\mathbf{J}(\lambda)} \Phi(\lambda, a) e^{-i \lambda a} g_{0}(a)+i g_{0}(a)
\end{aligned}
$$

and

$$
\begin{aligned}
\tilde{f}_{0}^{\prime}(a)= & \frac{1}{\mathbf{J}(\lambda)} J^{\prime}(\lambda, a) \int_{0}^{a} \Phi(\lambda, \xi) \rho(\xi) p(\xi)^{-1}\left((\lambda-2 i d(\xi)) g_{0}(\xi)-i g_{1}(\xi)\right) d \xi \\
& -\frac{i}{\mathbf{J}(\lambda)} \Phi^{\prime}(\lambda, a) e^{-i \lambda a} g_{0}(a)
\end{aligned}
$$

By the definition of the Jost solution, $J(\lambda, x)$ satisfies the boundary condition $i \lambda J(\lambda, a)+J^{\prime}(\lambda, a)=0$. So, to prove that $\left(\widetilde{f}_{0}^{\prime}+\widetilde{f}_{1}\right)(a)=0$ we have to show that

$$
\frac{\lambda}{\mathbf{J}(\lambda)} \Phi(\lambda, a) e^{-i \lambda a}+i=\frac{i}{\mathbf{J}(\lambda)} \Phi^{\prime}(\lambda, a) e^{-i \lambda a} .
$$

We recall $([19,20])$, that the equation $L_{\lambda} u=0$ has two linearly independent solutions, $J(\lambda, x)$ and $\widetilde{J}(\lambda, x)$. These solutions are uniquely defined by the following conditions:

$$
J(\lambda, x) e^{i \lambda x}=1 \quad \text { and } \quad \widetilde{J}(\lambda, x) e^{-i \lambda x}=1 \quad \text { for } \quad x \geq a .
$$

In terms of $J(\lambda, x)$ and $\widetilde{J}(\lambda, x)$ the solution $\Phi(\lambda, x)$ can be represented as

$$
\Phi(\lambda, x)=\frac{1}{2 i \lambda}[\widetilde{C}(\lambda) J(\lambda, x)+C(\lambda) \widetilde{J}(\lambda, x)],
$$

where the coefficients have the form $\widetilde{C}(\lambda)=\{\Phi, \widetilde{J}\}, C(\lambda)=-\{\Phi, J\}$. (By the curly brackets we denote the Wronskians of the corresponding functions.) It follows from 
(4.5) that $C(\lambda)=\mathbf{J}(\lambda)$, and, therefore, (4.35) can be written as

$$
\Phi(\lambda, x)=(2 i \lambda)^{-1}[\widetilde{C}(\lambda) J(\lambda, x)+\mathbf{J}(\lambda) \widetilde{J}(\lambda, x)] .
$$

From (4.35) and (4.36) we have

$$
\Phi(\lambda, a)=(2 i \lambda)^{-1}\left[\widetilde{C}(\lambda) e^{-i \lambda a}+\mathbf{J}(\lambda) e^{i \lambda a}\right], \quad \Phi^{\prime}(\lambda, a)=1 / 2\left[-\widetilde{C}(\lambda) e^{-i \lambda a}+\mathbf{J}(\lambda) e^{i \lambda a}\right] .
$$

Eq. (4.34) can be verified from (4.37) by a straightforward computation.

Now we prove statement b). Let us denote

$$
G_{0}(x)=\left[g_{0}(x)(\lambda-2 i d(x))-i g_{1}(x)\right] \mathcal{X}_{a} .
$$

In terms of $G_{0}$, the vector $\mathcal{R}_{\lambda}(\mathcal{B}) G$ can be written as

$$
\left(\mathcal{R}_{\lambda}(\mathcal{B}) G\right)(x)=\left(\begin{array}{c}
\left(L_{\lambda}^{-1} G_{0}\right)(x)-i[\mathbf{J}(\lambda)]^{-1} \Phi(\lambda, x) e^{-i \lambda a} g_{0}(a) \\
i \lambda\left(L_{\lambda}^{-1} G_{0}\right)(x)+\lambda[\mathbf{J}(\lambda)]^{-1} \Phi(\lambda, x) e^{-i \lambda a} g_{0}(a)+i g_{0}(x)
\end{array}\right) .
$$

Applying the operator $\left(A_{1}-\lambda I\right)$ to the two-component vector (4.39), we obtain the vector $\widetilde{F}=\left(\begin{array}{c}\widetilde{f}_{0} \\ \widetilde{f}_{1}\end{array}\right)$ having the following components:

$$
\begin{gathered}
\widetilde{f}_{0}(x)=g_{0}(x), \\
\widetilde{f}_{1}(x)=\left(L_{0} L_{\lambda}^{-1} G_{0}\right)(x)-i \lambda[\mathbf{J}(\lambda)]^{-1} e^{-i \lambda a} g_{0}(a)\left(L_{0} \Phi\right)(x) \\
+i \lambda(2 i d(x)-\lambda)\left(L_{\lambda}^{-1} G_{0}\right)(x)+\lambda[\mathbf{J}(\lambda)]^{-1} e^{-i \lambda a} \\
\times(2 i d(x)-\lambda) g_{0}(a) \Phi(\lambda, x)+i(2 i d(x)-\lambda) g_{0}(x) .
\end{gathered}
$$

Using (4.7) and (4.10), we collect the first and the third terms on the right of Eq. (4.40) and reduce them to the form

$$
\left(i L_{0}-2 \lambda d-i \lambda^{2}\right) L_{\lambda}^{-1} G_{0}=i L_{\lambda} L_{\lambda}^{-1} G_{0}=i G_{0} .
$$

Collecting the second and fourth terms on the right of Eq. (4.40), we obtain

$$
[\mathbf{J}(\lambda)]^{-1} g_{0}(a) e^{-i \lambda a}\left[L_{0}-\lambda^{2} I+2 i \lambda d(x)\right] \Phi(\lambda, x)=0,
$$

because $\Phi(\lambda, x)$ is an eigenfunction of $L_{\lambda}$. Substituting (4.41) and (4.42) into (4.40), we obtain

$$
\widetilde{f}_{1}(x)=G_{0}(x)+i(2 i d(x)-\lambda) g_{0}(x)=g_{1}(x),
$$

and (4.32) is shown. The proof is complete.

\section{ACKNOWLEDGEMENTS}

Partial support by the National Science Foundation Grant \#9212037 and by the Advanced Research Program - 93 of Texas Grant \#0003644-116 is gratefully acknowledged. 


\section{REFERENCES}

1. S. Cox, E. Zuazua, Rate at which energy decays in a damped string, Commun. in PDE, 19, n. 1/2, (1994), 213-243. MR 95f:35129

2. C.L. DeVito, Functional Analysis and Linear Operator Theory, Addison-Wesley Publishing Co., 1990. MR 91:47001

3. N. Dunford and J.T. Schwartz, Linear Operators, Part III, Spectral Operators, Wiley Interscience, 1971. MR 54:1009

4. B. Sz.-Nagy and C. Foias, Harmonic Analysis of Operators in Hilbert Spaces, Rev. ed., NorthHolland, Amsterdam, Holland, 1970. MR 43:947

5. I. Ts. Gohberg and M.G. Krein, Introduction to the Theory of Linear Nonselfadjoint Operators, Amer. Math. Soc., Providence, 1969. MR 39:7447

6. K. Hoffman, Banach Spaces of Analytic Functions, Prentice Hall, Englewood Cliffs., N.J., 1962. MR 24:A2844

7. T. Kato, Peturbation Theory for Linear Operators, Springer-Verlag, Berlin, 1976. MR 53:11389

8. P. Lax and R. Phillips, Scattering theory, rev. ed., Academic Press, New York, 1989. MR 90k:35005

9. P. Lax and R. Phillips, Scattering theory for dissipative hyperbolic systems, J. of Funct. Analysis 14 (1973), 172-235. MR 50:5502

10. P. Lax and R. Phillips, On the scattering frequencies of the Laplace operator for exterior domains, Comm. Pure Appl. Math., 25 (1972), 85-101. MR 45:5531

11. A.S. Markus, Introduction to the Spectral Theory of Polynomial Operator Pencils, Amer. Math. Soc., Providence, 1988. MR 89h:47023

12. R. Newton, Scattering Theory of Waves and Particles, 2nd Ed., Springer-Verlag, New York, 1980. MR 84f: 81001

13. N.K. Nikol'skii, Treatise on the Shift Operator, Springer-Verlag, 1985. MR 87i:47042

14. M.A. Pekker (Marianna A. Shubov), Nonphysical Sheet for the String Equation, J. Soviet Math., 10 (1978), 918-933. MR 56:734 (Russian original)

15. D. Russell, Nonharmonic Fourier series in the control theory of distributed parameter systems, J. Math. Anal. Appl., 18 (1967), 542-559. MR 35:1926

16. D. Russell, A unified boundary controllability theory for hyperbolic and parabolic partial differential equations, Studies in Appl. Math., 52 (1973), 189-211. MR 49:6006

17. D. Russell, Controllability and stabilizability theory for linear partial differential equations: recent progress and open questions, SIAM Review, 20 (1978), 639-739. MR 80c:93032

18. M.A. Shubov, Asymptotic of resonances and geometry of resonance states in the problem of scattering of acoustical waves by a spherically symmetric inhomogeneity of the density, Int. and Dif. Eq., 8 (1995), 1073-1115. MR 96a:47016

19. M.A. Shubov, Basis property of eigenfunctions of nonselfadjoint operator pencils generated by the equation of nonhomogeneous damped string, Integral Eq. Oper. Theory, 25 (1996), 289-328. MR 97e:47085

20. M.A. Shubov, Asymptotics of resonances and eigenvalues for nonhomogeneous damped string, Asymptotic Analysis, 13 (1996), 31-78. CMP 1996\#17

21. M.A. Shubov, Spectral decomposition method for controlled damped string. Reduction of control time. To appear in Applicable Analysis.

22. M.A. Shubov, Transformation operators for a class of damped hyperbolic equations, Preprint, Texas Tech. Univ., Lubbock, Texas, 1996.

23. M.A. Shubov, Spectral operators generated by damped hyperbolic equations. To appear in Integral Eq. Oper. Theory.

24. M.A. Shubov, C. Martin, J. Dauer and B. Belinskiy, Unique controllability of damped wave equation, to appear in SIAM Journal on Control and Optimization.

Department of Mathematics, Texas Tech University, Lubbock, Texas, 79409-1042

E-mail address: mshubov@math.ttu.edu 